\title{
Generalised Differential Quadrature Method in the Study of Free Vibration Analysis of Monoclinic Rectangular Plates
}

\author{
Prag Singhal",Garima Bindal
}

Department of Applied Sciences and Humanities, ABES Engineering College, Ghaziabad

\begin{abstract}
Vibration characteristics of monoclinic rectangular plate of exponentially varying thickness resting on elastic foundation have been studied on the bas is of classical plate theory. Following Lévy approach i.e. two parallel edges $(y=0$ and $b$ ) are assumed to be simply-supported while the other two edges ( $x=0$ and $a$ ) may have either of three co mbinations $\mathrm{C}$-C, C-S or C-F, where C, S and F stand for clamped, simply supported and free edge, respectively. Assuming the transverse displacement $w$ to vary as $\sin (p \pi y / b)$, the part ial diffe rential equation wh ich governs the motion of equation is reduced to an ordinary differential equation in $x$ with variable coefficients. The resulting ordinary differential equation has been solved by Generalised Differential Quadrature Method (GDQM) for all the boundary conditions considered here. The effect of various plate parameters has been studied on the natural frequencies for the first three modes of vibration. Convergence studies have been carried out for four decimal exactitude. Mode shapes for all the three plates have been presented. The efficiency of generalized differential quadrature method for the natural frequencies of vibration of monoclin ic rectangular plates has been examined.
\end{abstract}

Keywords GDQM, Monoclinic Material, Vibrations, Winkler Foundation

\section{Introduction}

Free vibration analys is of plate structure is one of the main vital tasks for an engineer to accomplish in the engineering design. Such type of free vibration problems are generally described by a linear partial differential equation associated with a set of related boundary conditions, whose closed form solution is not possible. As a result, the various numerical methods such as the finite difference method[1], Galerkin's method[2], Rayleigh-Ritz method[3],quintic splines method[4], finite-element method[5], Chebyshev collocation method[6], and Differential quadrature method[7-10] have been employed to study the vibrational characteristics of plates of various geometries.

Differential quadrature method (DQM) is a collocation scheme and was first introduced by Bellman et al.[11]. Malik and Civan[12] have presented comprehensive results showing that the DQM stands out in numerical accuracy as well as computational efficiency over the finite difference and finite element methods. The application of DQM covers almost all the areas of structural and vibration analys is of shells, beams and plates etc. The DQM is used to determine

Corresponding author:

prag.singhal@gmail.com (Prag Singhal)

Published online at http://journal.sapub.org/ajcam

Copyright (C) 2012 Scientific \& Academic Publishing. All Rights Reserved the weighting coefficients for the first order derivative approximation, in which the derivative of a function with respect to a spatial variable at a given discrete point can be expressed as a weighted linear sum of the function values at all the discrete points in the computational domain. Bellman et al.[13] proposed two methods to compute the weighting coefficients. In the first method, weighting coefficients are determined by solving a system of algebraic equations, in which the coordinate of the grid points to be chosen arbitrary. But, in this method, it is very difficult to obtain the weighting coefficients for a large number of grid points as it results to an ill-conditioned matrix since the corresponding differential matrix becomes increasingly large [14]. In the second method, the weighting coefficients are determined by a simple algebraic formula and the grid points are chosen as the roots of shifted Legendre polynomial. Shu et a1.[15] proposed the generalized differential quadrature (GDQ) method in which Lagrange interpolation polynomial is used as the bas is function. An algebraic expression is presented to compute the weighting coefficients of the first order derivative approximation and the recurrence formula is used to compute the weighting coefficients for higher order derivatives without restriction on the choice of the grid points.

The advantages of the GDQ method included no restriction on the number of grid points used for the approximation and the weighted coefficients are determined 
using simple recurrence relation instead of solving a set of linear algebraic equations as in other version of differential quadrature method. A more in depth analys is of the merits of the GDQ method can be found in Du. et al.[16]. Recently, the GDQ method has been applied to solve fluid dynamics and buckling of composite structure problems $[17,18]$.

The plates of variable thic kness have significantly greater efficiency for vibration as compared to the plates of uniform thickness and also provide the advantage of reduction in weight and size. Notable contribution made thereafter dealing with rectangular plates with uniform / non-uniform thickness with various boundary conditions are given in the refs. [19-23].

The analysis of structures on elastic foundations is of considerable interest and widely used in several engineering fields, such as foundation, pavement and railroad, pipeline and some aero-space structures applications. The different foundation models (Vlasov, Winkler and Pasternak etc.) have been proposed in the literature [24] to appro ximate the foundation characteristics. Numerous studies dealing with Winkler foundation are available in the literature and are reported in the refs. $[25,26]$.

The free vibration analysis of homogeneous monoclinic rectangular plates with exponentially varying thickness resting on Winkler foundation has been solved by Generalized Differential Quadrature method (GDQM). Frequencies and mode shapes for different boundary conditions (C-C, C-S and C-F) for the first three modes of vibration are computed. The present work and the results may be an orientation to the future research work.

\section{Mathematical Formulation}

Consider a rectangular monoclinic plate of length a, breadth $\mathrm{b}$, thickness $\mathrm{h}$, density $\rho$ and resting on a Winkler-type foundation with foundation modulus $k_{f}$ referred to rectangular Cartesian co-ordinates system. The differential equation governing the free transverse vibration of monoclinic rectangular plate is given by

$$
\begin{aligned}
& c_{11} \frac{h^{3}}{12} \frac{\partial^{4} w}{\partial x^{4}}+c_{22} \frac{h^{3}}{12} \frac{\partial^{4} w}{\partial y^{4}}+2\left(c_{12}+c_{21}+c_{66}\right) \frac{h^{3}}{12} \frac{\partial^{4} w}{\partial x^{2} \partial y^{2}} \\
& +2 \frac{\partial}{\partial x}\left\{\left(c_{12}+c_{21}+c_{66}\right) \frac{h^{3}}{12}\right\} \frac{\partial^{3} w}{\partial x \partial y^{2}}+2 \frac{\partial}{\partial y}\left\{\left(c_{12}+c_{21}+c_{66}\right)\right. \\
& \left.\frac{h^{3}}{12}\right\} \frac{\partial^{3} w}{\partial y \partial x^{2}}+2 \frac{\partial}{\partial x}\left\{c_{11} \frac{h^{3}}{12}\right\} \frac{\partial^{3} w}{\partial x^{3}}+2 \frac{\partial}{\partial y}\left\{c_{22} \frac{h^{3}}{12}\right\} \frac{\partial^{3} w}{\partial y^{3}} \\
& +\frac{\partial^{2}}{\partial x^{2}}\left\{c_{11} \frac{h^{3}}{12}\right\} \frac{\partial^{2} w}{\partial x^{2}}+\frac{\partial^{2}}{\partial y^{2}}\left\{c_{22} \frac{h^{3}}{12}\right\} \frac{\partial^{2} w}{\partial y^{2}} \\
& +\frac{\partial^{2}}{\partial y^{2}}\left\{\left(c_{12}+c_{21}\right) \frac{h^{3}}{12}\right\} \frac{\partial^{2} w}{\partial x^{2}}+\frac{\partial^{2}}{\partial x^{2}}\left\{\left(c_{12}+c_{21}\right) \frac{h^{3}}{12}\right\} \frac{\partial^{2} w}{\partial y^{2}} \\
& +4 \frac{\partial^{2}}{\partial y \partial x}\left(c_{66} \frac{h^{3}}{12}\right\} \frac{\partial^{2} w}{\partial y \partial x}+\rho h \frac{\partial^{2} w}{\partial t^{2}}+K_{f} w=0
\end{aligned}
$$

where $\mathrm{w}(\mathrm{x}, \mathrm{y}, \mathrm{t})$ is the transverse deflection, $\mathrm{t}$ the time, $\rho$ the mass density, $c_{11}, c_{12}, c_{21}, c_{22}$ and $c_{66}$ are the material constants.

Let us assume that two opposite edges of the plate given by $\mathrm{y}=0$ and $\mathrm{y}=\mathrm{b}$ are simply-supported and that the thickness is independent of y i.e., $h=h(x)$. For harmonic vibrations, the deflection function $\mathrm{w}$ (Levy approach) is assumed to be

$$
w(x, y, t)=\bar{w}(x) \sin (p \pi y / b) e^{i \omega t}
$$

where ' $\mathrm{p}$ ' is the positive integer and $\omega$ the circular frequency in radians.

By substituting (2) in (1) and introducing the following non-dimensional variables

$$
X=x / a, Y=y / b, \bar{h}=h / a, w=\bar{w} / a
$$

The equation (1) reduces to

$$
\begin{aligned}
& \bar{h}^{3} w^{i v}(X)+6 \bar{h}^{2} \bar{h}^{\prime} w^{\prime \prime}(X)+\left\{6 \bar{h} \bar{h}^{2}+3 \bar{h}^{2} \bar{h}^{\prime \prime}-\right. \\
& \left.2 \frac{\left(c_{12}+c_{21}+c_{66}\right)}{c_{11}} \lambda^{2} \bar{h}^{3}\right\} w^{\prime \prime}(X)-6 \frac{\left(c_{12}+c_{21}+c_{66}\right)}{c_{11}} \\
& \lambda^{2} \bar{h}^{2} \bar{h} w^{\prime}(X)+\left\{\frac{c_{22}}{c_{11}} \lambda^{4} \bar{h}^{3}-3 \frac{\left(c_{12}+c_{21}\right)}{c_{11}}\left(2 \bar{h} \bar{h}^{2}+\bar{h}^{2} \bar{h}^{\prime \prime}\right) \lambda^{2}\right. \\
& \left.-\frac{12 \rho \bar{h} a^{2} \omega^{2}}{c_{11}}+\frac{12 a K_{f}}{c_{11}}\right\} w(X)=0
\end{aligned}
$$

where $\lambda^{2}=\frac{p^{2} a^{2} \pi^{2}}{b^{2}}$ and primes denote the differentiation w.r.t. ' $X$ '.

We as sume that the thickness and density of the plate are varying exponentially along the X-direction and are given by $\bar{h}=h_{o} e^{\alpha X}$ and $\rho=\rho_{o} e^{\beta X}$, where $h_{o}$ and $\rho_{o}$ are the thickness and density of the plate respectively at the end $X=0$, $\alpha$ and $\beta$ are the taper and density parameters respectively.

With these notations, equation (3) reduces to

$$
\begin{aligned}
& A_{o} w^{i v}(X)+A_{1} w^{\prime \prime \prime}(X)+A_{2} w^{\prime \prime}(X)+A_{3} w^{\prime}(X) \\
& +A_{4} w(X)=0
\end{aligned}
$$

where

$$
\begin{aligned}
& A_{o}=1, A_{1}=6 \alpha, A_{2}=9 \alpha^{2}-2 \frac{\left(c_{12}+c_{21}+c_{66}\right)}{c_{11}} \lambda^{2}, \\
& A_{3}=-6 \frac{\left(c_{12}+c_{21}+c_{66}\right)}{c_{11}} \lambda^{2} \alpha, \\
& A_{4}=\frac{c_{22}}{c_{11}} \lambda^{4}-9 \frac{\left(c_{12}+c_{21}\right)}{c_{11}} \lambda^{2} \alpha^{2}-\Omega^{2} e^{(\beta-2 \alpha) X}+ \\
& \frac{12 a K_{f} e^{-3 \alpha X}}{c_{11} h_{o}^{3}}, \Omega^{2}=\frac{12 \rho a^{2} \omega^{2}}{c_{11} h_{o}^{2}}
\end{aligned}
$$

The solution of equation (4) together with the boundary conditions at the edges $X=0$ and $X=1$ gives rise to a two-point boundary value problem with variable coeffic ients whose closed form solution is not possible. An approximate solution is obtained by employing generalised differential quadrature method.

\section{Method of solution: Generalised}




\section{Differential Quadrature Method}

The GDQ approximates a partial derivative of a function with respect to a variable at any discrete point as a weighted sum of the function values at all the discrete points chosen in the overall domain of that variable and approximates continuous function with a higher-order polynomial in the overall variable domain. The $n^{\text {th }}$ order derivative of $W(X)$ w.r.t. $X$ can be expressed discretely at the point $X_{\mathrm{i}}$ as

$$
\frac{d^{n} W\left(X_{i}\right)}{d X^{n}}=\sum_{j=1}^{m} c_{i j}^{(n)} W\left(X_{j}\right)
$$

$\mathrm{n}=1,2,3,4$ and $\mathrm{i}=1,2, \ldots, m$

where $m$ is the total number of grid points employs to discretize the plate, $c_{i j}^{(n)}$ are the weighting coefficients associated with the nth order derivative of $\mathrm{W}(X)$ w. r. t. $X$ at discrete point $X_{\mathrm{i}}$. In the generalized differential quadrature (GDQ) method $[16,17]$, the global Lagrange interpolation polynomial is used as the test function

$$
g_{j}(X)=\frac{M(X)}{\left(X-X_{j}\right) M^{(1)}\left(X_{j}\right)}, \mathrm{j}=1,2, \ldots, \mathrm{m} ;
$$

where

$$
\left.\begin{array}{c}
M(X)=\prod_{j=1}^{m}\left(X-X_{j}\right) \\
M^{(1)}\left(X_{i}\right)=\prod_{\substack{j=1 \\
j \neq i}}^{m}\left(X_{i}-X_{j}\right)
\end{array}\right\}
$$

where $M^{(1)}(X)$ is the first derivative of $M(X)$. Thus, the weighting coefficients $c_{i j}^{(1)}(\mathrm{i}, \mathrm{j}=1,2, \ldots, \mathrm{m})$ can be obtained analytically fro $m$ the diffe rentiation of equation (6) to obtain

$$
\begin{gathered}
c_{i j}^{(1)}=\frac{M^{(1)}\left(X_{i}\right)}{\left(X_{i}-X_{j}\right) M^{(1)}\left(X_{j}\right)}, \\
i, j=1,2, \ldots, m ; i \neq j \\
c_{i i}^{(1)}=-\sum_{\substack{j=1 \\
j \neq i}}^{m} c_{i j}^{(n)}, \mathrm{i}=1,2, \ldots, m ;
\end{gathered}
$$

The weighting coefficients for higher order derivatives can be obtained in the same manner. Actually, a recurrence relationship has been found for the nth order weighting coefficients $c_{i j}^{(n)}$.

$$
\begin{array}{r}
c_{i j}^{(n)}=n\left(c_{i i}^{(n-1)} c_{i j}^{(1)}-\frac{c_{i j}^{(n-1)}}{x_{i}-x_{j}}\right) \\
i, j=1,2, \ldots, m, j \neq i \text { and } n=2,3,4 \\
c_{i i}^{(n)}=-\sum_{\substack{j=1 \\
j \neq i}}^{m} c_{i j}^{(n)}, i=1,2, \ldots, m ; n=1,2,3,4
\end{array}
$$

Discretizing equation (4) at grid points $X_{\mathrm{i}}, i=3,4, \ldots, m-2$, it reduces to,

$$
\begin{aligned}
& A_{0, i} W^{i v}\left(X_{i}\right)+A_{1, i} W^{\prime \prime \prime}\left(X_{i}\right)+A_{2, i} W^{\prime \prime}\left(X_{i}\right)+ \\
& A_{3, i} W^{\prime}\left(X_{i}\right)+A_{4, i} W\left(X_{i}\right)=0 .
\end{aligned}
$$

Substituting for $W(X)$ and its derivatives at the $i^{\text {th }}$ grid point in the equation (11) and using relations (5) to (10), the equation (11) becomes

$$
\begin{aligned}
& \sum_{j=1}^{m}\left(A_{0, i} c_{i j}^{(4)}+A_{1, i} c_{i j}^{(3)}+A_{2, i} c_{i j}^{(2)}+A_{3, i} c_{i j}^{(1)}\right) W\left(X_{j}\right)+ \\
& A_{4, i} W\left(X_{i}\right)=0 .
\end{aligned}
$$

For $i=3,4, \ldots,(m-2)$, one obtains a set of $(m-4)$ equations in terms of unknowns $W_{j}\left(\equiv W\left(X_{j}\right)\right), j=1,2, \cdots, m$, which can be written in the matrix form as

$$
[B]\left[W^{*}\right]=[0]
$$

where $B$ and $W^{*}$ are matrices of order $(m-4) \times m$ and $(m \times 1)$ respectively.

Here, the $(m-2)$ internal grid points chosen for collocation, are the zeros of shifted Chebyshev polynomial of order $(m-2)$ with orthogonality range $[0,1]$ given by

$$
X_{k+1}=\frac{1}{2}\left[1+\cos \left(\frac{2 k-1}{m-2} \frac{\pi}{2}\right)\right], k=1,2, \ldots, m-2
$$

\section{Boundary Conditions and Frequency Equations}

The three different combinations of boundary conditions namely, C-C, C-S and C-F have been considered here, where C, S, F stand for clamped, simply supported and free edge, respectively and first symbol denotes the condition at the edge $X=0$ and second symbol at the edge $X=1$. By satisfying the relations

$$
\begin{gathered}
W=\frac{d W}{d X}=0 \\
W=\frac{d^{2} W}{d X^{2}}-c_{11} \lambda^{2} W=0 ;
\end{gathered}
$$

and

$$
\frac{d^{2} W}{d X^{2}}-c_{11}^{\prime} \lambda^{2} W=0=\frac{d^{3} W}{d X^{3}}-\left(\frac{c_{11}^{\prime}}{c_{11}}+4 \frac{c_{66}}{c_{11}}\right) \lambda^{2} \frac{d W}{d X}=0,
$$

where $c_{11}^{\prime}=\left(\frac{c_{12}+c_{21}}{c_{11}}\right)$ for clamped, simply supported and free edge conditions, respectively, a set of four homogeneous equations in terms of unknown $W_{\mathrm{j}}$ are obtained. These equations together with field equation (13) give a complete set of $m$ homogeneous equations in $m$ unknowns. For C-C plate this set of equations can be written as

$$
\left[\begin{array}{l}
B \\
B^{C C}
\end{array}\right]\left[W^{*}\right]=[0]
$$

where $\mathrm{B}^{C C}$ is a matrix of order $4 \times m$. 
For a non-trivial solution of equation (15), the frequency determinant must vanish and hence,

$$
\left|\begin{array}{l}
B \\
B^{C C}
\end{array}\right|=0
$$

Similarly for C-S and C-F plates, the frequency determinants can be written as

$$
\left|\begin{array}{l}
B \\
B^{C S}
\end{array}\right|=0 \text { and }\left|\begin{array}{l}
B \\
B^{C F}
\end{array}\right|=0 \text { respectively }(17,18)
$$

\section{Numerical Results and Discussion}

The frequency equations (16-18) have been solved numerically to compute the values of the frequency parameter $\Omega$ for various values of plate parameters. The effect of foundation parameter, density parameter, taper parameter and aspect ratio on frequency parameter $\Omega$ has been analysed for C-C, C-S and C-F plates vibrating in first three modes of vibration. The elastic constants for the plate material (rock gypsum)[Haussuhl] are taken as

$$
\begin{aligned}
& c_{11}=7.859 \times 10^{6} \mathrm{erg} / \mathrm{cm}^{3}, c_{12}=c_{21}=4.1 \times 10^{6} \mathrm{erg} / \mathrm{cm}^{3}, \\
& c_{22}=6.287 \times 10^{6} \mathrm{erg} / \mathrm{cm}^{3}, c_{66}=1.044 \times 10^{6} \mathrm{erg} / \mathrm{cm}^{3},
\end{aligned}
$$

given by Haussuhl,1965 (rock gypsum) as monoclinic material. This is obtained by taking $\mathrm{p}=1$ and thickness $h_{0}$
$=0.1$ at the edge $X=0$.

To choose the appropriate number of collocation points $m$, convergence studies have been carried out for various sets of parameters for all the three plates. Convergence graphs are

shown in the figures $1(\mathrm{a}-\mathrm{c})$ for $a / b=1.0, K=0.02, \alpha=0.5$ and $\beta=0.5$ for C-C, C-S and C-F plates respectively. For these data the maximum deviation were observed. In all the computations, $m=20$ has been fixed for all the boundary conditions in the first three modes since further increase in $\mathrm{m}$ does not improve the results even in the fourth place of decimal.

The behaviour of frequency parameter $\Omega$ with density parameter $\beta$ for the first mode of vibration for $\mathrm{C}-\mathrm{C}, \mathrm{C}-\mathrm{S}$ and $\mathrm{C}-\mathrm{F}$ plates for two different values of foundation parameter $K=0.0,0.02$, taper parameter $\alpha=-0.5,0.5$ and aspect ratio $a / b=1$ is shown in figure 2(a). It is found that the frequency parameter $\Omega$ decreases with increasing values of density parameter $\beta$ keep ing other plate parameters fixed. The rate of decrease in frequency parameter $\Omega$ with $\beta$ increases with the increase in the values of foundation parameter $K$ and taper parameter $\alpha$. This rate of decrease is higher in the order C-C > $\mathrm{C}-\mathrm{S}>\mathrm{C}-\mathrm{F}$ plates. A similar behavior is observed for the second and third modes of vibration as shown in figures $2(\mathrm{~b})$ and 2(c). The rate of decrease in $\Omega$ with $\beta$ is higher in third mode as compared to second and first modes for all the plates.

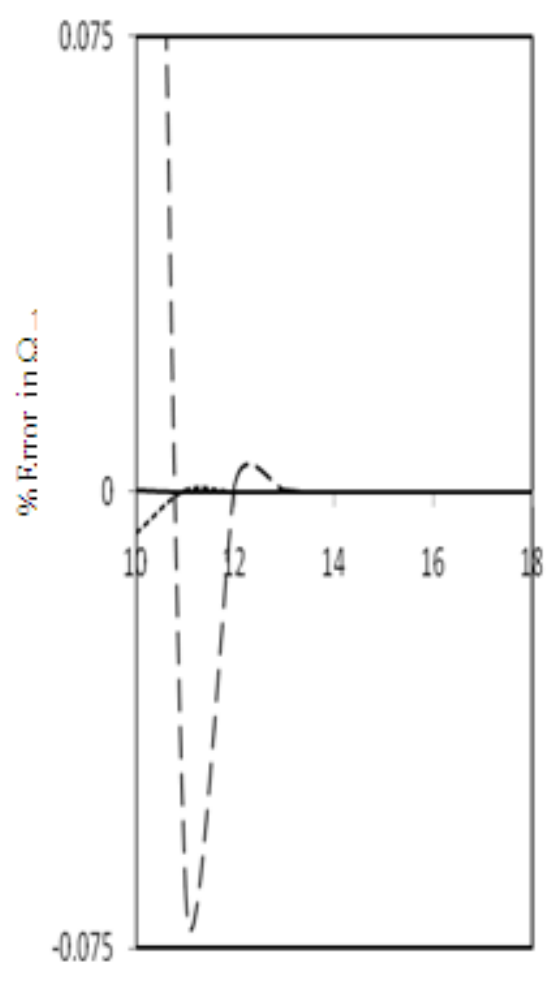

$\mathrm{m}$ (number of nodes) $\rightarrow$
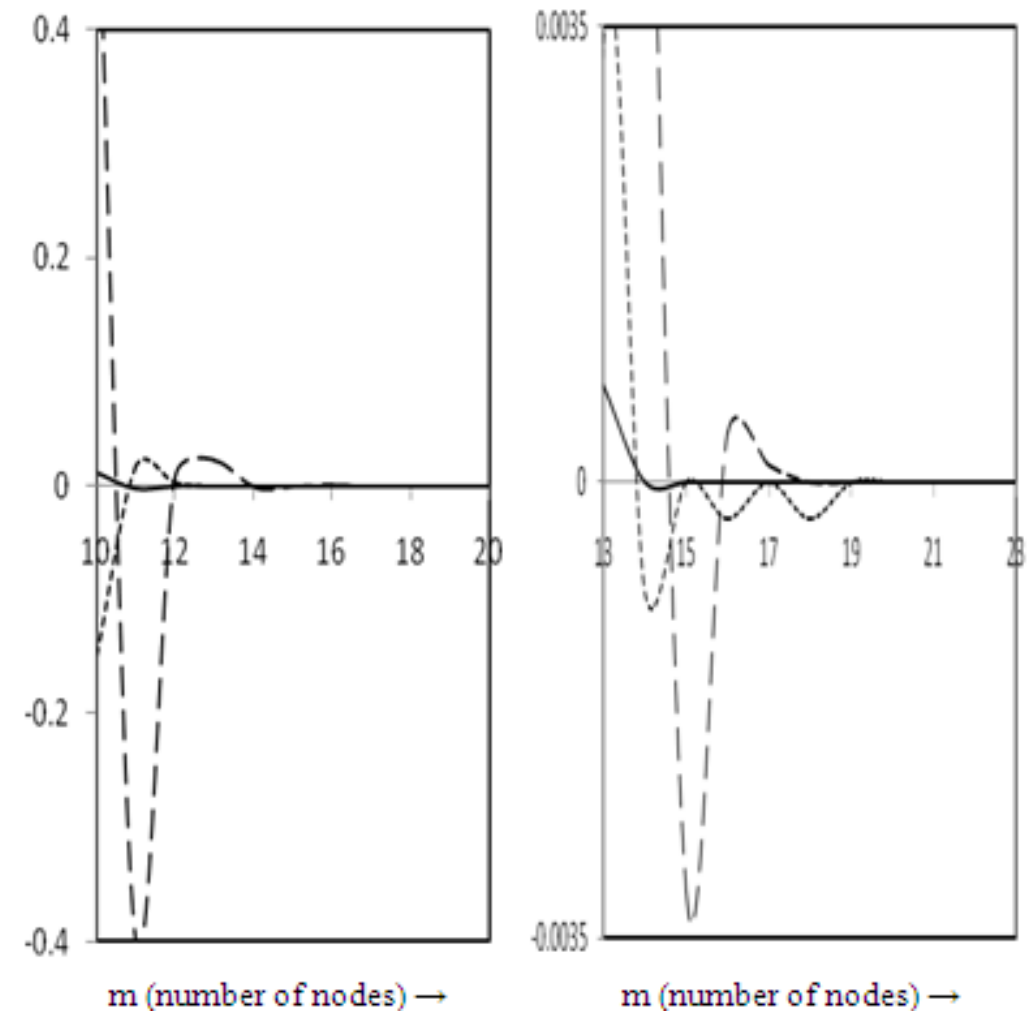

Figure 1. Percentage error in frequency parameter $\Omega$; (a) C-C plate, (b) C-S plate and (c) C-F plate, for $a / b=1.0, K=0.02, \beta=0.5, \alpha=0.5, \longrightarrow$, first mode,------, second mode, ----- , third mode. \%error $=\left[\left(\Omega_{\mathrm{m}}-\Omega_{20}\right) / \Omega_{20}\right] \times 100$ 
Figure 3(a) depicts the plots of frequency parameter $\Omega$ versus taper parameter $\alpha$ for two different values of $K=0.0$, $0.02, \beta=-0.5,0.5$ and $a / b=1$ for the first mode of vibration. From the graphs, it is observed that the frequency parameter $\Omega$ increases with the increasing values of taper parameter $\alpha$ in the absence of foundation parameter for all the three plates irrespective of other plate parameters. However, in the presence of foundation i.e. $K=0.02$, the behaviour for C-C and C-S plates remain same but in case of C-F plate for $K=$ $0.02, a / b=1$ and $\beta=-0.5,0.5$ the frequency parameter $\Omega$ first decreases and then increases with the increasing values of taper parameter $\alpha$. In particu lar for a C-F plate for $K=0.02$, $a / b=1$ and $\beta=-0.5,0.5$ it is found that there is a local minima in the vicinity of $\alpha=0.4$. In case of second and third mode of vibration figures $3(\mathrm{~b}) \& 3(\mathrm{c})$, the frequency parameter $\Omega$ increases with increasing values of taper parameter $\alpha$ for all the boundary conditions. The rate of increase for C-S plate is higher as compared to $\mathrm{C}-\mathrm{F}$ p late but smaller than that of C-C plate. This rate of increase in second mode of vibration is smaller as compared to third mode but greater than that of first mode for all the boundary conditions.
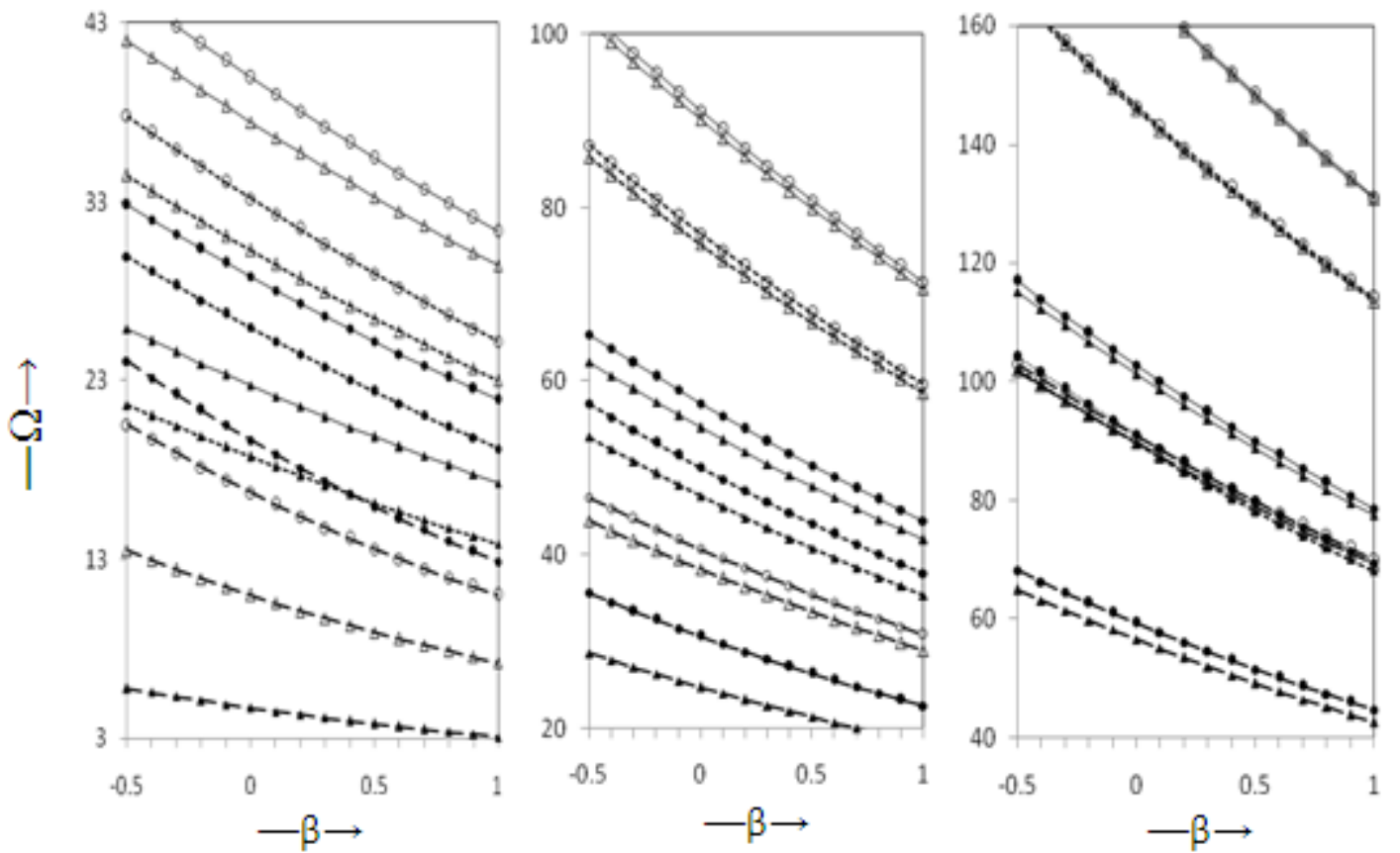

Figure 2. Frequency parameter for C-C and C-S plates: (a) First Mode, (b) Second mode, (c) Third Mode, for a/b $=1.0,-\longrightarrow$, C-C; ------, C-S; ---,C-F plates; $\mathbf{\square}, \alpha=-0.5, K=0.0, \square, \alpha=0.5, K=0.0, \downarrow, \alpha=-0.5, K=0.02, \diamond, \alpha=0.5, K=0.02$
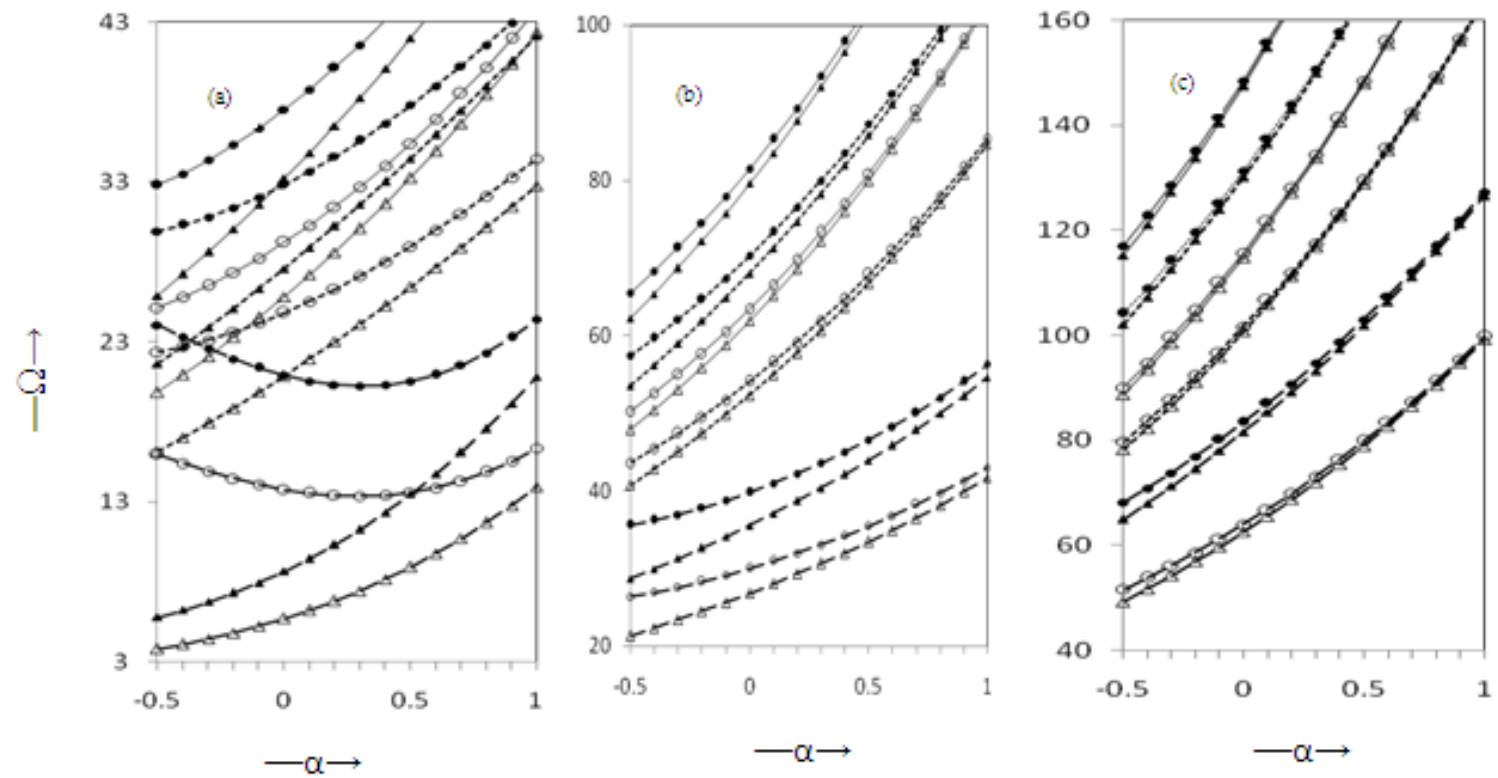

Figure 3. Frequency parameter for C-C and C-S plates: (a) First Mode, (b) Second mode, (c) Third Mode, for $a / b=1.0,-\longrightarrow$, C-C; ------, C-S; --- -, C-F plates; $\mathbf{\square}, \beta=-0.5, K=0.0, \square, \beta=0.5, K=0.0, \diamond, \beta=-0.5, K=0.02, \diamond, \beta=0.5, K=0.02$ 
The behavior of the aspect ratio $a / b$ on the frequency parameter $\Omega$ for the first mode of vibration for two different values of $K=0.0,0.02, \alpha=-0.5,0.5$ and $\beta=0.5$ is shown in the figure 4(a). It is found that the frequency parameter $\Omega$ increases with the increasing values of the aspect ratio $a / b$ for all the three plates. The rate of increase of frequency parameter $\Omega$ with aspect ratio $a / b$ decreases with the increasing values of foundation parameter $K$ while increases with the increasing values of taper parameter $\alpha$. A similar inference can be drawn from figures 4(b) \& 4(c) showing the plots for second and third modes of vibration. This rate of increase is higher in C-S plate as compared to C-C and C-F plate in the first three modes. This rate of increase in second mode of vibration is greater as compared to first mode but smaller than that of third mode for all the boundary conditions.

Figures 5(a) - 5(c) shows the plots of the frequency parameter $\Omega$ versus foundation parameter $K$ for density parameter $\beta=-0.5,0.5$, aspect ratio $a / b=0.5,1.0$ and taper parameter $\alpha=0.5$. It is observed that the frequency parameter $\Omega$ increases with the increasing values of $K$ for all the three boundary conditions. The rate of increase in frequency parameter $\Omega$ with foundation parameter $K$ is higher in case of C-S plate as compared to C-C plate but smaller than that of $\mathrm{C}-\mathrm{F}$ plate for the same set of values of other plate parameters. The rate of increase goes on decreasing with the increase in the order of modes.
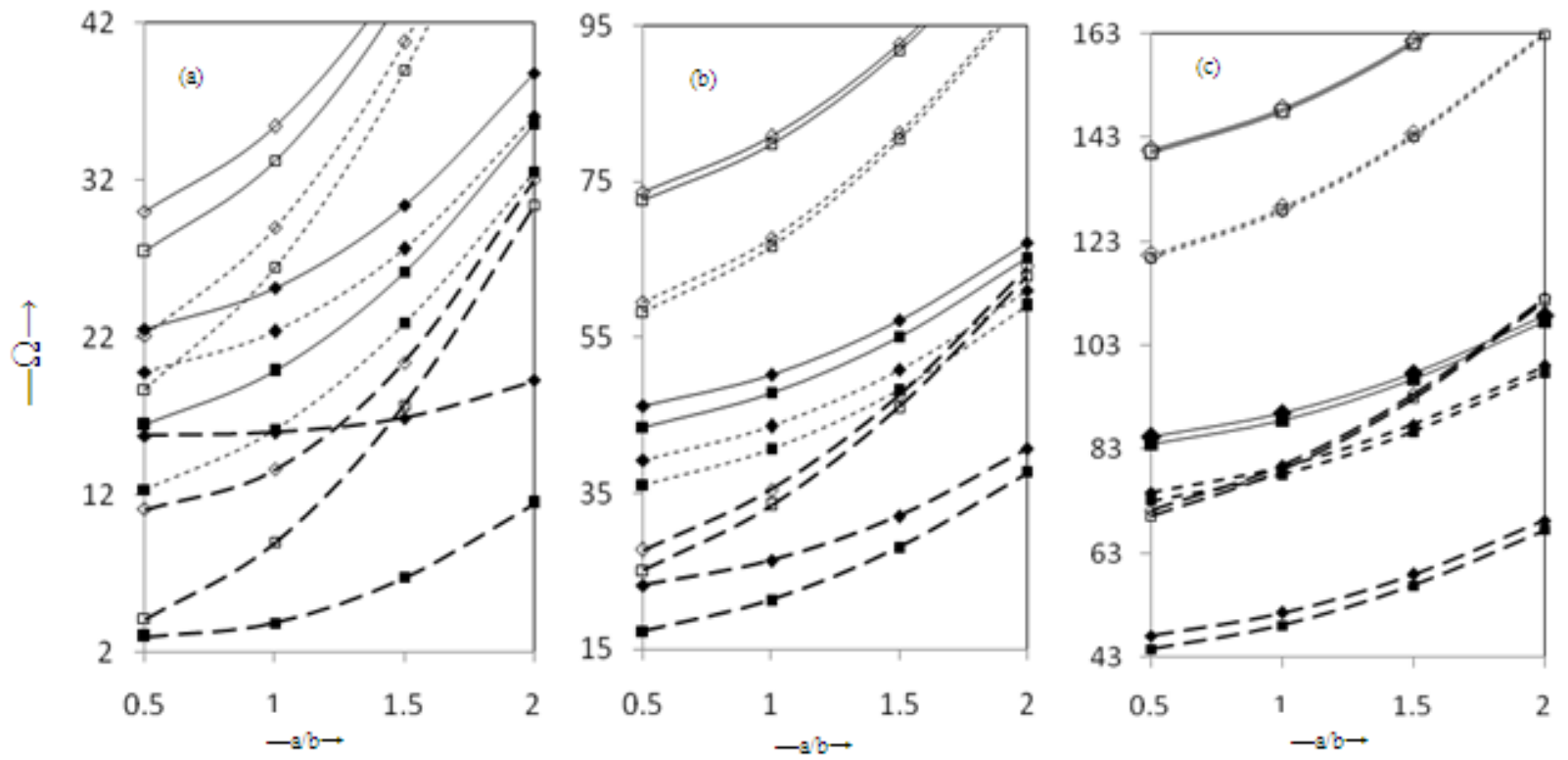

Figure 4. Frequency parameter for C-C and C-S plates: (a) First Mode, (b) Second mode, (c) Third Mode, for $\beta=0.5,-\longrightarrow, \mathrm{C}-\mathrm{C}$; - - - -, C-S;---, C-F plates; $\mathbf{\square}, \alpha=-0.5, K=0.0, \square, \alpha=0.5, K=0.0, \downarrow, \alpha=-0.5, K=0.02, \diamond, \alpha=0.5, K=0.02$
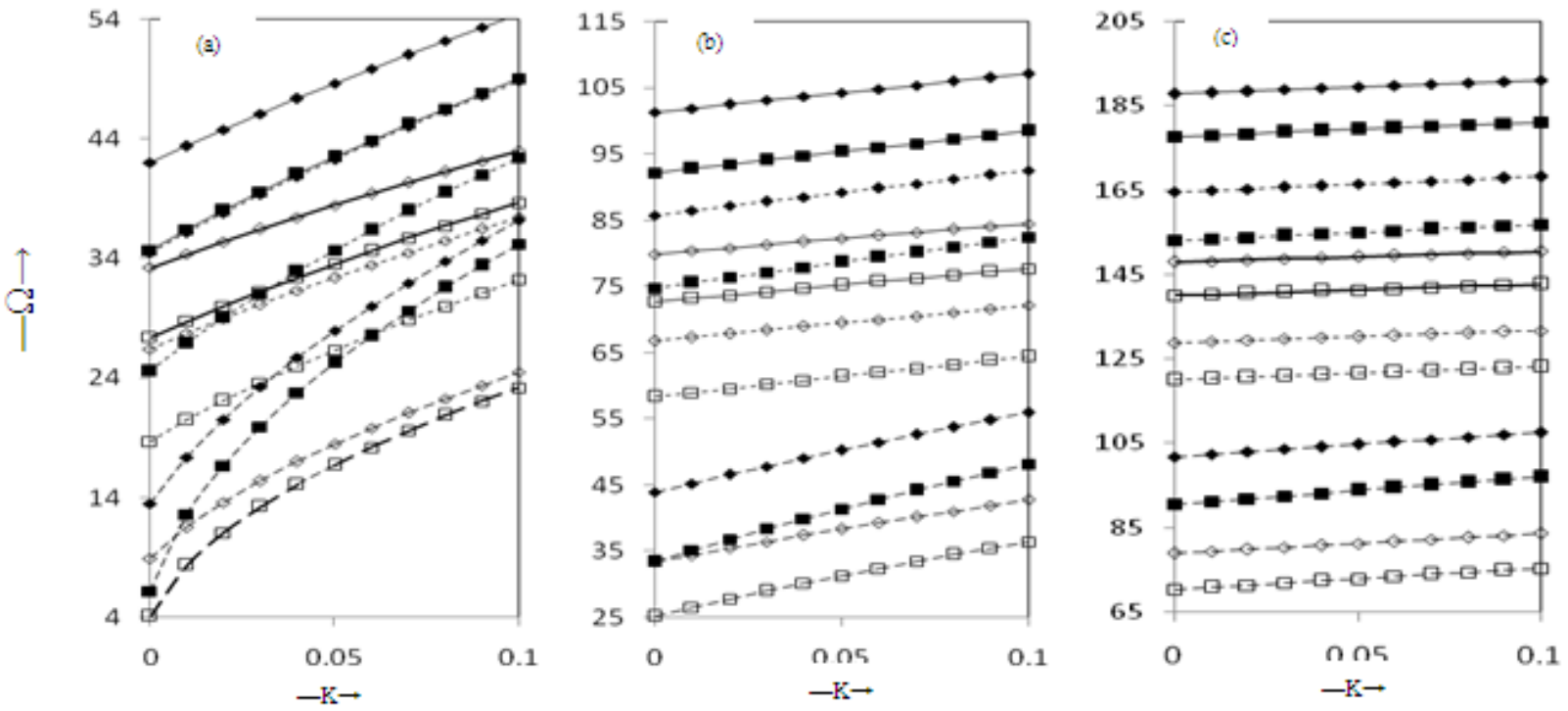

Figure 5. Frequency parameter for C-C and C-S plates: (a) First Mode, (b) Second mode, (c) Third Mode, for $\alpha=0.5,-\longrightarrow$, C-C; -----, C-S; ;---,C-F plates; $\mathbf{\square}, a / b=-0.5, \beta=-0.5, \square, a / b=-0.5, \beta=0.5, \bullet, a / b=1.0, \beta=-0.5, \diamond, a / b=1.0, \beta=0.5$ 

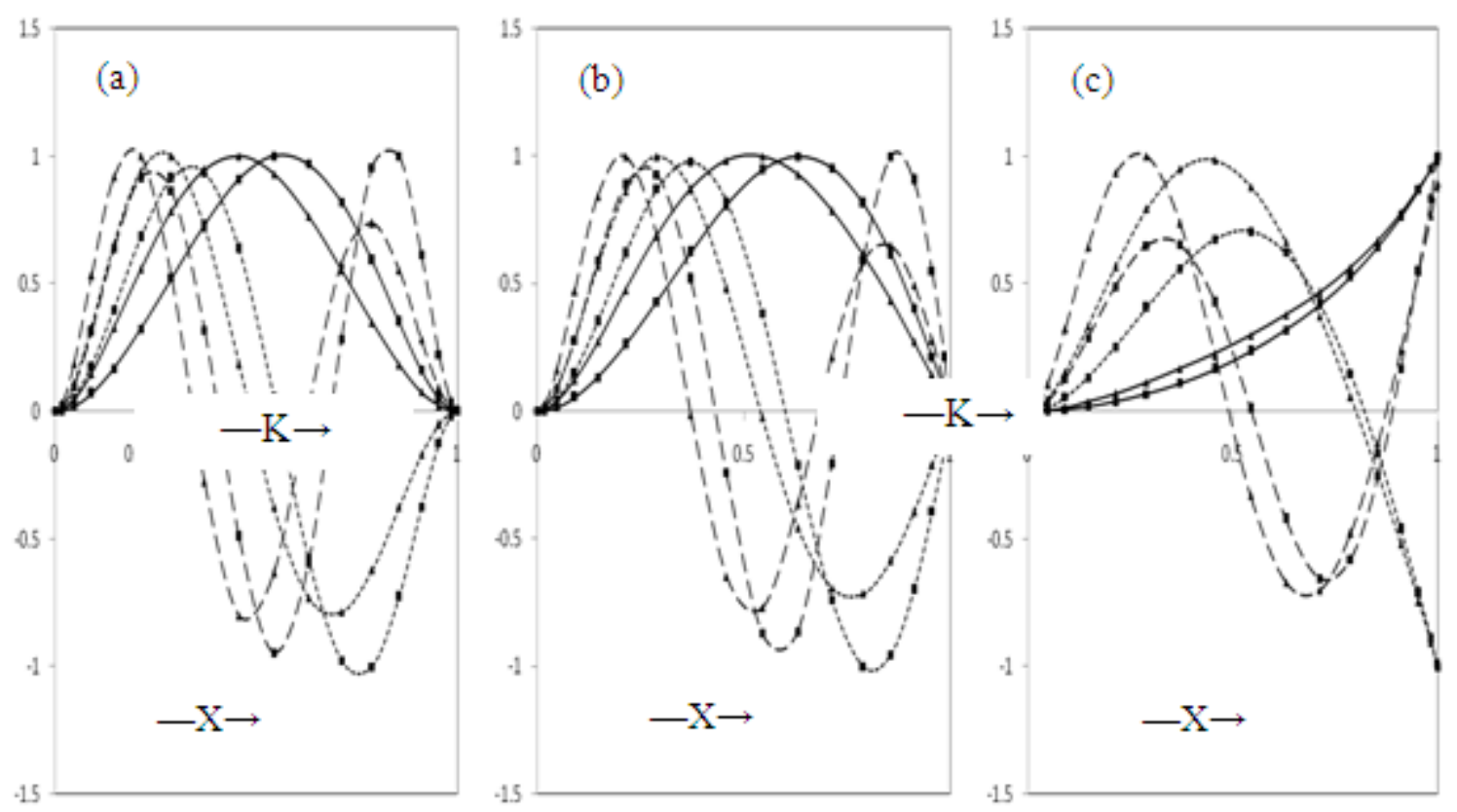

Figure 6. Normal displacements: (a) C-C plate, (b) C-Splate, (c) C-C plate, for $a / b=1.0, \quad \beta=0.5, K=0.02$, --- , third mode; $\mathbf{\square}, \alpha=-0.5 ; \boldsymbol{\Delta}, \alpha=0.5$

Figures 6(a) - 6(c) shows the plots for normalized transverse displacements for a square plate i.e $a / b=1.0$ and $K=0.02, \beta=0.5, \alpha=-0.5,0.5$ for the first three mode of vibration for clamped, simply supported and free plate, respectively. The nodal lines are found to shift towards the edge $X=1$ as $\alpha$ increases from -0.5 to 0.5 i.e as the plate becomes thicker at outer edge.

\section{Conclusions}

The effects of Winkler foundation on natural frequencies of rectangular plates composed by a monoclinic material with exponentially varying thickness have been studied on the basis of classical plate theory. It is observed that frequency parameter $\Omega$ increases with the increase in foundation parameter $K$ and aspect ratio $a / b$ while decreases with the increasing value of density para meter $\beta$ keeping all other plate parameters fixed for all the three boundary conditions. However, the behaviour with taper parameter $\alpha$ is not monotonous.

The implementation of the GDQ method is relatively easier and the efforts needed for solving the problem with GDQM is also relatively less in comparison to the other numerical methods. The limitations of the proposed method are that the accuracy does not meet in case of complicated geometries.

The present study is of great significance to design engineers in obtaining the desired frequency by a proper choice of one or more parameters considered here.

\section{REFERENCES}

[1] B. Singh, S.M. Hassan, J. Lal, "Some numerical experiments on high accuracy fast direct finite difference methods for elliptic problem", Communications in Numerical Methods in Engineering, vol.2, pp.631-641, 1996.

[2] D.R. Avalos, H. Hack, P.A.A. Laura, "Galerkin's method and axisymmetric vibrations of polar orthotropic circular plates", AIAA Journal, vol.20, pp.1626-1628, 1982.

[3] U.S. Gupta, A.H. Ansari, "Transverse vibration of polar orthotropic parabolically tapered circular plates", Indian Journal of Pure and Applied Mathematics, vol.34, no.6, pp.819-830, 2003.

[4] R.Lal, U.S.Gupta, Reena, "Quintic Splines in the study of Transverse vibrations of non- uniform Orthotropic rectangular plates", Jl. of Sound and Vibration, vol. 207, no.1, pp.1-13, 1997

[5] D.V. Chen, B.S. Ren, "Finite element analysis of the lateral vibration of thin annular and circular plates with variable thickness", ASME Journal of Vibration and Acoustics, vol.120, no.3, pp.747-752, 1998.

[6] Chebyshev collocation method in the study of Transverse vibrations of non-uniform rectangular orthotropic plates", The Shock and Vibration Digest, vol.33, no.2, pp.103-112, 2001.

[7] P. Malekzadeh and S.A. Shahpari, "Free vibration analy sis of variable thickness thin and moderately thick plates with elastically restrained edges by DQM", Thin-Walled Structures, vol.43, no.7, pp.1037-1050, 2005.

[8] U.S. Gupta, R. Lal and S. Sharma, "Vibration analysis of non-homogeneous circular platr of non-linear thickness variation by differential quadrature method", Journal of Sound and Vibration, vol.298, no.4-5, pp.892-906, 2006.

[9] R. Lal, Dhanpati and Y. Kumar, "Buckling and vibration of non-homogeneous orthotropic rectangular plates of varying thickness under biaxial compression", International $\mathrm{Jl}$. of applied Math and Mechanics, vol.4, no.4, pp.93-107, 2008. 
[10] Prag, "Free transverse vibrations of non-uniform composite plates", Ph. D Thesis, Gurukul Kangri University Hardwar, U.K, India, 2012.

[11] R.E. Bellman, J. Casti, "Differential quadrature and long term integration”, J. Math Anal. Appl., vol.34, pp.235-238, 1971.

[12] M. Malik, and F. Civan, “A comparative study of differential quadrature and cubature method vis-à-vis some conventional techniques in context of convection-diffusion-reaction problems", Chemical Engineering Science, vol.50, no.1, pp.531-547, 1995.

[13] Bellman, B.G. Kashef, J. Casti, "Differential quadrature: a technique for the rapid solution of non-linear partial differential equations", J. Comp. Phys., vol.10, pp.40-52, 1972.

[14] C. Shu, "Differential quadrature and its application in Engineering", Springer, New York, 2000.

[15] C. Shu, B.E. Richard, “Application of generalized differential quadrature to solve two -dimensions incompressible Navier-stress equations", Int. J. Num. Meth. Fluids, vol.15, pp.791-798, 1992.

[16] H. Du, M.K. Lim and R.M. Lin , “Application of gen eralized differential quadrature Du method to structural problems", International Journal for Numerical Method in Engineering, vol.37, no.1, pp.1881-1896, 1994.

[17] C.Shu and H. Du, “A generalized approach for implementing general boundary conditions in the generalized differential quadrature, free vibration analysis of plates", International Journal of Solid and Structures, 1995.

[18] M. Fatahi and A. Shokuky, "Analysis of delamination buckling and post buckling of composite structure by generalized differential quadrature method", International Journal of Advanced Engineering Sciences and Technologies, vol.1, no.1, pp.30-37, 2010.

[19] D.V. Bambill, C.A. Rossit, P.A.A. Laura, R.E. Rossi, "Transverse vibration of an orthotropic rectangular plate of linearly varying thickness and with a free edge", Journal of Sound and Vibration, vol.235, no.3, pp.530-538, 2000.

[20] R. Lal, U.S. Gupta, C. Goel, "Chebyshev polynomials in the study of transverse vibrations of non-uniform rectangular orthotropic plates", The Shock and Vibration Digest, vol.33, no.2, pp.103-112, 2001.

[21] S. Hurlebaus, L. Gaul, J.T.-S. Wang, "An exact series solution for calculating the eigen frequencies of orthotropic plates with completely free boundary", Journal of Sound and Vibration, vol.244, no.5, pp.747-759, 2001.

[22] M. Huang, X.Q. Ma, T. Sakiyama, H. Matuda, C. Morita, "Free vibration analy sis of orthotropic rectangular plates with variable thickness and general boundary conditions", Journal of Sound and Vibration, vol.288, no.4-5, pp.931-955, 2005.

[23] R. Lal, Y. Kumar and U.S. gupta, "Transverse vibration of non-homogeneous rectangular plates of uniform thickness using boundary characteristic orthogonal polynomials", Int. J1. of Appl. Math and Mechanics, vol. 6. No.14,pp.93-109, 2010

[24] Selvadurai APS., "Elastic Analysis of Soil-Foundation Interaction", Elsevier, NY, 1979.

[25] R. Lal and Dhanpati, "Transverse vibrations of non-homogeneous orthotropic rectangular plates of variable thickness: A spline technique", Journal of Sound and Vibration, vol.306, no.1-2, pp.203-214, 2007.

[26] M.F. Liu, T.P. Chang and Y.H. Wang, "Free vibration analy sis of orthotrop ic rectan gular plates with tapered vary ing thickness and Winkler spring foundation”, Machine Based Design of Structures and Machines: An International Journal, vol.39, no.3, pp.320-333, 2011. 